\title{
On multivariate Wilson bases
}

Bownik, Marcin; Jakobsen, Mads Sielemann; Lemvig, Jakob; Okoudjou, Kasso A.

Published in:

Proceedings of 2017 International Conference on Sampling Theory and Applications

Link to article, DOI:

10.1109/SAMPTA.2017.8024456

Publication date:

2017

Document Version

Peer reviewed version

Link back to DTU Orbit

Citation (APA):

Bownik, M., Jakobsen, M. S., Lemvig, J., \& Okoudjou, K. A. (2017). On multivariate Wilson bases. In Proceedings of 2017 International Conference on Sampling Theory and Applications (pp. 192-5). IEEE. https://doi.org/10.1109/SAMPTA.2017.8024456

\section{General rights}

Copyright and moral rights for the publications made accessible in the public portal are retained by the authors and/or other copyright owners and it is a condition of accessing publications that users recognise and abide by the legal requirements associated with these rights.

- Users may download and print one copy of any publication from the public portal for the purpose of private study or research.

- You may not further distribute the material or use it for any profit-making activity or commercial gain

- You may freely distribute the URL identifying the publication in the public portal

If you believe that this document breaches copyright please contact us providing details, and we will remove access to the work immediately and investigate your claim. 


\section{On Multivariate Wilson Bases}

\author{
Marcin Bownik \\ Department of Mathematics \\ University of Oregon \\ Eugene, OR 97403-1222, USA \\ E-mail: mbownik@uoregon.edu
}

Jakob Lemvig

Technical University of Denmark

Department of Applied Mathematics and Computer Science

Matematiktorvet 303B

2800 Kgs. Lyngby, Denmark

E-mail: jakle@dtu.dk

\author{
Mads S. Jakobsen \\ Norwegian University of Science and Technology \\ Department of Mathematical Sciences \\ Trondheim, Norway \\ E-mail: mads.jakobsen@ntnu.no \\ Kasso A. Okoudjou \\ Department of Mathematics \\ University of Maryland \\ College Park \\ MD 20742, USA \\ E-mail: kasso@math.umd.edu
}

\begin{abstract}
A Wilson system is a collection of finite linear combinations of time frequency shifts of a square integrable function. In this paper we give an account of the construction of bimodular Wilson bases in higher dimensions from Gabor frames of redundancy two.
\end{abstract}

\section{INTRODUCTION}

Daubechies, Jaffard and Journé [4], inspired by work of K. G. Wilson [11], introduced in 1991 the Wilson system of (linear combinations of) time-frequency shifts of a univariate function $g \in L^{2}(\mathbb{R})$ :

$$
\begin{aligned}
\mathcal{W}_{1}(g) & =\left\{T_{n} g\right\}_{n \in \mathbb{Z}} \\
& \cup\left\{\frac{1}{\sqrt{2}} T_{n}\left(M_{m}+(-1)^{m} M_{-m}\right) g\right\}_{n \in \mathbb{Z}, m \in \mathbb{N}} \\
& \cup\left\{\frac{1}{\sqrt{2}} T_{n+1 / 2}\left(M_{m}-(-1)^{m} M_{-m}\right) g\right\}_{n \in \mathbb{Z}, m \in \mathbb{N}},
\end{aligned}
$$

where the translation operator $T_{\lambda}$ and the modulation operator $M_{\gamma}$ are given by

$$
T_{\lambda} f(x)=f(x-\lambda), \quad M_{\gamma} f(x)=e^{2 \pi i x \cdot \gamma} f(x),
$$

for $f \in L^{2}\left(\mathbb{R}^{d}\right)$ and $\lambda, \gamma \in \mathbb{R}^{d}$. The main result in [4] is a construction of Wilson orthonormal bases generated by functions $g \in L^{2}(\mathbb{R})$ with good time and frequency localization. This should be compared with the Gabor system $\left\{M_{\gamma} T_{\lambda} g\right\}_{\lambda \in \Lambda, \gamma \in \Gamma}$, where such basis constructions are impossible for any pair $\Lambda$ and $\Gamma$ of full-rank lattices in $\mathbb{R}^{d}$. Indeed, the Balian-Low Theorem [1], [2], [9] states that if a Gabor system is a orthonormal basis or a Riesz basis for $L^{2}\left(\mathbb{R}^{d}\right)$, then the generating window function $g$ cannot have rapid decay in time and frequency.

However, it is easy to construct "nice" window functions $g$ generating redundant Gabor frames. The main result of [4] illustrates how this fact can be used to construct nice orthonormal Wilson bases for $L^{2}(\mathbb{R})$ :
Theorem I.1 ([4]). Let $g \in L^{2}(\mathbb{R})$ be such that $\hat{g}(\omega)=\overline{\hat{g}(\omega)}$ and $\|g\|_{2}=1$. Then the Gabor system $\left\{M_{m} T_{n / 2} g\right\}_{m, n \in \mathbb{Z}}$ is a tight frame for $L^{2}(\mathbb{R})$ with frame bound $A=2$ if, and only if, the Wilson system $\mathcal{W}_{1}(g)$ is an orthonormal basis for $L^{2}(\mathbb{R})$.

From the definition (1), it is clear that, except from the pure translations $\left\{T_{n} g\right\}_{n \in \mathbb{Z}}$, the univariate Wilson systems produce a bimodular covering of the frequency line, in the sense that each element of the system has two peaks in its power spectrum $|\hat{g}|^{2}$, assuming the window function is sufficiently localized in frequency. On the other hand, Gabor systems are unimodular since each element has a single peak in the power spectrum. In many applications a bimodular covering of the frequency domain is as good as a unimodular covering, in particular, if the signals of interest are realvalued.

As an example of an application of Theorem I.1, we mention that the continuous, symmetric function $g(x)=\cos (\pi x) \mathbb{1}_{[-1 / 2,1 / 2]}(x)$ with compact support generates a tight Gabor frame $\left\{M_{m} T_{n / 2} g\right\}_{m, n \in \mathbb{Z}}$ with frame bound $A=2$ and $\|g\|_{2}=1$. Thus, the Wilson system $\mathcal{W}(g)$ is an orthonormal basis for $L^{2}(\mathbb{R})$.

The construction of Wilson orthonormal bases has recently been generalized to higher dimensions $L^{2}\left(\mathbb{R}^{d}\right)$ by the authors in [10]. The aim of this note is to give an overview of these results.

\section{CONSTRUCTION OF MULTIVARIATE BIMODULAR WILSON BASES}

In higher dimensions, it is obviuously possible to construct orthonormal Wilson bases for $L^{2}\left(\mathbb{R}^{d}\right)$ by taking tensor products of univariate window functions from Theorem I.1. However, tensoring Wilson bases to $L^{2}\left(\mathbb{R}^{d}\right)$ has several undesirable side effects: 
a) the basis functions of tensored Wilson basis are $2^{d}$-modular hence they give rise to a $2^{d}$-modular covering of the frequency domain,

b) tensored Wilson bases are associated with highly redundant Gabor frames of redundancy $2^{d}$,

c) the generating function of a tensored Wilson basis has to be a separable function of the form $g_{1}\left(x_{1}\right) \ldots g_{d}\left(x_{d}\right)$.

Gabor frames $\left\{M_{\gamma} T_{\lambda} g\right\}_{\lambda \in \Lambda, \gamma \in \Gamma}$ are unimodular in all dimensions, hence give rise to a unimodular covering of the frequency domain. The $2^{d}$-modular coverings are a curse of dimensionality of tensored Wilson bases since, e.g., symmetric peaks of the power spectrum of real-valued signals will leak out to $2^{d-1}$ other locations in frequency.

Our goal in this paper is to construct Wilson orthonormal bases in higher dimension that do not suffer from these tensoring artifacts.

\section{A. Setup and Notation}

We use the following assumptions throughout the paper: We let $N$ be a subset of $\mathbb{Z}^{d}$ such that $N \cap$ $(-N)=\emptyset$ and $N \cup(-N) \cup\{0\}=\mathbb{Z}^{d}$. For $g \in L^{2}\left(\mathbb{R}^{d}\right)$ we consider the Gabor system ${ }^{1}$

$$
\mathcal{G}(g)=\left\{T_{\lambda} M_{\gamma} g\right\}_{\lambda \in \Lambda, \gamma \in \mathbb{Z}^{d}},
$$

where $\Lambda:=\mathbb{Z}^{d} \cup\left(\mathbf{1} / \mathbf{2}+\mathbb{Z}^{d}\right)$ and where we use boldface $\mathbf{1} / \mathbf{2}$ to denote the constant vector $(1 / 2, \ldots, 1 / 2) \in$ $\mathbb{R}^{d}$, and the Wilson system

$$
\begin{aligned}
\mathcal{W}(g) & =\left\{T_{\lambda} g\right\}_{\lambda \in \mathbb{Z}^{d}} \\
& \cup\left\{\frac{1}{\sqrt{2}} T_{\lambda}\left(M_{\gamma}+(-1)^{|\gamma|} M_{-\gamma}\right) g\right\}_{\lambda \in \mathbb{Z}^{d}, \gamma \in N} \\
& \cup\left\{\frac{1}{\sqrt{2}} T_{\lambda+\frac{1}{\mathbf{2}}}\left(M_{\gamma}-(-1)^{|\gamma|} M_{-\gamma}\right) g\right\}_{\lambda \in \mathbb{Z}^{d}, \gamma \in N},
\end{aligned}
$$

where $(-1)^{|n|}$ means $(-1)^{n_{1}+n_{2}+\ldots+n_{d}}$ for vectors $n \in \mathbb{Z}^{d}$. For any dimension $d \in \mathbb{N}$ the Wilson system $\mathcal{W}(g)$, as opposed to tensored Wilson systems, provides a bimodular covering of the frequency domain for localized windows with the two peaks in frequency being symmetric about the origin. A priori it is not clear that this is the correct definition of Wilson systems in $L^{2}\left(\mathbb{R}^{d}\right)$, in particular, as there are many more symmetries possible in higher dimensions. The results of the next subsection will justify the definition of $\mathcal{W}(g)$.

\section{B. Main result}

We have the following duality principles of Gabor frames and Wilson bases.

${ }^{1}$ This convention is a slight abuse of language since the defined system is only unitarily equivalent with the Gabor system $\left\{M_{\gamma} T_{\lambda} g\right\}$. However, these systems share all frame theoretic properties, and we will not make any distinction between such systems in the remainder of this paper.
Theorem II.1 ([10]). Suppose that $\hat{g}(\omega)=\overline{\hat{g}(\omega)}$. Then the following holds:

(i) The Gabor system $\mathcal{G}(g)$ is a frame with bounds $2 A$ and $2 B$ for $L^{2}\left(\mathbb{R}^{d}\right)$ if and only if the Wilson system $\mathcal{W}(g)$ is a Riesz basis with bounds $A$ and $B$ for $L^{2}\left(\mathbb{R}^{d}\right)$.

(ii) The Gabor system $\mathcal{G}(g)$ is a tight frame for $L^{2}\left(\mathbb{R}^{d}\right)$ with frame bound $A=2$ if and only if the Wilson system $\mathcal{W}(g)$ is an orthonormal basis for $L^{2}\left(\mathbb{R}^{d}\right)$.

The "only if"-assertion in (i) is Corollary 8.5.6 in [5] for $d=1$, albeit without bounds. Assertion (ii) of Theorem II.1 generalizes Theorem I.1 to higher dimensions in a non-tensored way.

In the following example we construct bimodular multivariate Wilson bases from Gabor frames of redundancy two. The construction follows the standard construction procedure of "nice" generators $g$ of univariate Wilson bases, see e.g., [4].

Example 1. Let $g \in L^{2}\left(\mathbb{R}^{d}\right)$ be a window function in the Wiener space $W\left(\mathbb{R}^{d}\right)$ satisfying the symmetry condition $\hat{g}(\omega)=\overline{\hat{g}(\omega)}$ and

$$
\underset{x, \omega \in[0,1)^{d}}{\operatorname{essinf}}\left(|Z g(x, \omega)|^{2}+\left|Z g\left(x-\frac{\mathbf{1}}{\mathbf{2}}, \omega\right)\right|^{2}\right)>0,
$$

where $Z$ denotes the Zak transform. By [5, Theorem 8.3.1], it follows that the Gabor system $\left\{T_{\lambda} M_{\gamma} g\right\}_{\lambda \in \mathbb{Z}^{d} \cup\left(\mathbf{1} / \mathbf{2}+\mathbb{Z}^{d}\right), \gamma \in \mathbb{Z}^{d}}$ is a frame. Let $S$ denote the Gabor frame operator. Define $h=S^{-1 / 2} g=$ $Z^{-1} q Z g$, where $q=\left(|Z g|^{2}+\left|Z T_{\mathbf{1} / \mathbf{2}} g\right|^{2}\right)^{-1 / 2} \in$ $L^{\infty}\left([0,1)^{2}\right)$. Since the action of the frame operator preserves the symmetry of $g$, we conclude that $\left\{T_{\lambda} M_{\gamma} h\right\}_{\lambda \in \mathbb{Z}^{d} \cup\left(\mathbf{1} / \mathbf{2}+\mathbb{Z}^{d}\right), \gamma \in \mathbb{Z}^{d}}$ is a Parseval frame whose window function satisfies $\hat{h}(\omega)=\overline{\hat{h}(\omega)}$. An application of Theorem II.1 yields that the Wilson system generated by $\sqrt{2} h \in W\left(\mathbb{R}^{d}\right)$ is an orthonormal basis for $L^{2}\left(\mathbb{R}^{d}\right)$.

\section{Auxiliary results}

The following simple relationship between frame operators of the Gabor system and the Wilson system in Proposition II.2 seems not to have been noticed before in the literature.

Proposition II.2 ([10]). Suppose that $\hat{g}(\omega)=\overline{\hat{g}(\omega)}$. Then the Gabor system $\mathcal{G}(g)$ is a Bessel sequence with bound $B$ if and only if the Wilson system $\mathcal{W}(g)$ is a Bessel sequence with bound B/2. Furthermore, in either (and hence both cases) the Gabor frame operator $S_{\mathcal{G}}$ and the Wilson frame operator $S_{\mathcal{W}}$ satisfy

$$
S_{\mathcal{G}}=2 S_{\mathcal{W}}
$$

The following density-type theorem for Wilson system is an easy consequence of Theorem II.1 and Proposition II.2. 
Corollary II.3. If $\mathcal{W}(g)$ is a frame for $L^{2}\left(\mathbb{R}^{d}\right)$ with bounds $A$ and $B$, then $\mathcal{W}(g)$ is a Riesz basis for $L^{2}\left(\mathbb{R}^{d}\right)$ with bounds $A$ and $B$.

Proof. If $\mathcal{W}(g)$ is a frame for $L^{2}\left(\mathbb{R}^{d}\right)$ with bounds $A$ and $B$, then, by Proposition II.2, so is $\mathcal{G}(g)$ with bounds $2 A$ and $2 B$. The conclusion now follows from Theorem II.1(i).

\section{Proof TEChNiQues}

The proof of the results in Section II from [10] are mostly based on the frame theory of shift-invariant systems [3], [6], [7]. We illustrate in this section how this theory can be applied in the study of Wilson bases. We refer to [10] for a detailed argumentation and a proof of Theorem II.1.

\section{A. Frame theory of shift-invariant system}

Definition III.1. Let $\Gamma$ be a countable index set and let $\left\{g_{\gamma}\right\}_{\gamma \in \Gamma} \subset L^{2}\left(\mathbb{R}^{d}\right)$. For a full-rank lattice $\Lambda=P \mathbb{Z}^{d}$, where $P \in \mathrm{GL}_{d}(\mathbb{R})$, the dual lattice is given by $\Lambda^{\perp}=$ $\left(P^{-1}\right)^{\top} \mathbb{Z}^{d}$. Suppose that

$$
\sum_{\gamma \in \Gamma}\left|\hat{g}_{\gamma}(\omega)\right|^{2}<\infty \quad \text { for a.e. } \omega \in \mathbb{R}^{d} .
$$

For the shift-invariant system $\left\{T_{\lambda} g_{\gamma}\right\}_{\lambda \in \Lambda, \gamma \in \Gamma}$ we define its autocorrelation functions $\left\{t_{\alpha}\right\}_{\alpha \in \Lambda^{\perp}}$ by

$$
t_{\alpha}(\omega):=\frac{1}{|\operatorname{det} P|} \sum_{\gamma \in \Gamma} \hat{g}_{\gamma}(\omega) \overline{\hat{g}_{\gamma}(\omega-\alpha)}
$$

for a.e. $\omega \in \mathbb{R}^{d}$ and $\alpha \in \Lambda^{\perp}$.

For a given function $t \in L^{\infty}\left(\mathbb{R}^{d}\right)$, define the multiplication operator

$$
M_{t} f(x)=t(x) f(x) \quad \text { for } f \in L^{2}\left(\mathbb{R}^{d}\right) .
$$

For the special choice of $t(x)=e^{2 \pi i\langle x, \gamma\rangle}$ with $\gamma \in \mathbb{R}^{d}$, this yields the modulation operator $M_{\gamma}$, justifying our notation.

Lemma III.2 ([6], [7]). Let $K=P \mathbb{Z}^{d}$, where $P \in$ $\mathrm{GL}_{d}(\mathbb{R})$, be a full-rank lattice, let $\Gamma$ be a countable index set, and let $\left\{g_{\gamma}\right\}_{\gamma \in \Gamma} \subset L^{2}\left(\mathbb{R}^{d}\right)$. For the shiftinvariant system $\left\{T_{\lambda} g_{\gamma}\right\}_{\lambda \in K, \gamma \in \Gamma}$ the followings holds:

(i) $\left\{T_{\lambda} g_{\gamma}\right\}_{\lambda \in K, \gamma \in \Gamma}$ is a tight frame for $L^{2}\left(\mathbb{R}^{d}\right)$ with frame bound $A$ if, and only if, for all $\alpha \in K^{\perp}$,

$$
t_{\alpha}(\omega)=A \delta_{\alpha, 0}
$$

for almost all $\omega \in \mathbb{R}^{d}$. In either case, the series defining $t_{\alpha}$ are a.e. absolutely convergent and $\left|t_{\alpha}(\omega)\right| \leq A$ for a.e. $\omega \in \mathbb{R}^{d}$.

(ii) If $\left\{T_{\lambda} g_{\gamma}\right\}_{\lambda \in K, \gamma \in \Gamma}$ is a Bessel sequence with bound $B$, then the series (4) defining $t_{\alpha}$ are a.e. absolutely convergent, $\left|t_{\alpha}(\omega)\right| \leq B$ for a.e. $\omega \in \mathbb{R}^{d}$, and the frame operator $S$ has a Walnut representation:

$$
\widehat{S f}=\sum_{\alpha \in K^{\perp}} M_{t_{\alpha}} T_{\alpha} \hat{f}
$$

with unconditionally norm convergence for all $\hat{f} \in L^{\infty}\left(\mathbb{R}^{d}\right)$ with compact support.

B. On autocorrelation functions of Wilson and Gabor systems

Recall that $\Lambda:=\mathbb{Z}^{d} \cup\left(\mathbf{1} / \mathbf{2}+\mathbb{Z}^{d}\right)$. One readily verifies that $\Lambda$ is a full-rank lattice with its dual lattice given by

$$
\Lambda^{\perp}=\left\{n \in \mathbb{Z}^{d}: n_{1}+n_{2}+\ldots+n_{d} \in 2 \mathbb{Z}\right\} .
$$

The usefulness of the frame theory of shift-invariant systems in the construction of Wilson bases is the following key lemma.

Lemma III.3 ( [10]). Let $g \in L^{2}\left(\mathbb{R}^{d}\right)$ and $\hat{g}(\omega)=$ $\overline{\hat{g}(\omega)}$. Suppose that

$$
\sum_{\gamma \in \mathbb{Z}^{d}}|\hat{g}(\omega-\gamma)|^{2}<\infty
$$

for a.e. $\omega \in \mathbb{R}^{d}$. Then the following holds:

(i) If the Gabor system $\mathcal{G}(g)$ is considered as a shiftinvariant system with generators $\left\{M_{\gamma} g\right\}_{\gamma \in \mathbb{Z}^{d}}$ and with shifts along the lattice $\Lambda=\mathbb{Z}^{d} \cup\left(\mathbf{1} / \mathbf{2}+\mathbb{Z}^{d}\right)$, then its autocorrelation functions are given by

$$
t_{\alpha, \mathcal{G}}(\omega)=2 \sum_{\gamma \in \mathbb{Z}^{d}} \hat{g}(\omega-\gamma) \overline{\hat{g}(\omega-\gamma-\alpha)},
$$

for $\alpha \in \Lambda^{\perp}$, a.e. $\omega \in \mathbb{R}^{d}$.

(ii) If the Wilson system $\mathcal{W}(g)$ is considered as a shift-invariant system with generators

$$
\begin{array}{r}
g,\left\{\frac{1}{\sqrt{2}}\left(M_{\gamma}+(-1)^{|\gamma|} M_{-\gamma}\right) g\right\}_{\gamma \in N} \\
\text { and }\left\{\frac{1}{\sqrt{2}} T_{\mathbf{1} / \mathbf{2}}\left(M_{\gamma}-(-1)^{|\gamma|} M_{-\gamma}\right) g\right\}_{\gamma \in N}
\end{array}
$$

and with shifts along the lattice $\mathbb{Z}^{d}$, then its autocorrelation functions are given by, for $\alpha \in \Lambda^{\perp}$,

$$
t_{\alpha, \mathcal{W}}(\omega)=\sum_{\gamma \in \mathbb{Z}^{d}} \hat{g}(\omega-\gamma) \overline{\hat{g}(\omega-\gamma-\alpha)},
$$

and for $\alpha \in \mathbb{Z}^{d} \backslash \Lambda^{\perp}, t_{\alpha, \mathcal{W}}(\omega)=0$ for a.e. $\omega \in$ $\mathbb{R}^{d}$.

Sketch of the proof. Assumption (6) guarantees that generators of $\mathcal{G}(g)$ and $\mathcal{W}(g)$ satisfy condition (3). Hence, their autocorrelation functions are well-defined. It is straightforward to verify (i) from (4).

In the following we sketch the proof of (ii) for $d=1$. In dimension one, we find $\Lambda=(1 / 2) \mathbb{Z}, \Lambda^{\perp}=2 \mathbb{Z}$; the canonical choice of the set $N$ is $\mathbb{N}$, but we will allow 
a general index set $N$. By Definition III.1, for $\alpha \in \mathbb{Z}$ we have

$$
\begin{aligned}
t_{\alpha, \mathcal{W}}(\omega)= & \hat{g}(\omega) \overline{\hat{g}(\omega-\alpha)} \\
& +\frac{1}{2} \sum_{\gamma \in N} G(--)+(-1)^{|\gamma|} G(-+) \\
& \quad+(-1)^{|\gamma|} G(+-)+G(++) \\
& +\frac{1}{2}(-1)^{\alpha} \sum_{\gamma \in N} G(--)-(-1)^{|\gamma|} G(-+) \\
& \quad-(-1)^{|\gamma|} G(+-)+G(++) .
\end{aligned}
$$

where $G( \pm \pm):=\hat{g}(\omega \pm \gamma) \overline{\hat{g}(\omega \pm \gamma-\alpha)}$. Due to the phase factor $(-1)^{\alpha}$, we will consider two cases: (I) $\alpha \in 2 \mathbb{Z}$, and (II) $\alpha \in 2 \mathbb{Z}+1$, corresponding to $\alpha \in \Lambda^{\perp}$ and $\alpha \in \mathbb{Z}^{d} \backslash \Lambda^{\perp}$, respectively. Using that $N \cup(-N) \cup$ $\{0\}=\mathbb{Z}$ and $N,-N$ and $\{0\}$ are mutually disjoint sets, formula (7) yields:

(I) for $\alpha \in \Lambda^{\perp}=2 \mathbb{Z}$

$$
t_{\alpha, \mathcal{W}}(\omega)=\sum_{\gamma \in \mathbb{Z}} G(--)
$$

(II) for $\alpha \in \mathbb{Z} \backslash \Lambda^{\perp}=\mathbb{Z} \backslash 2 \mathbb{Z}$

$$
t_{\alpha, \mathcal{W}}(\omega)=\sum_{\gamma \in \mathbb{Z}}(-1)^{|\gamma|} G(-+)
$$

Note that the symmetry property $\hat{g}(\omega)=\overline{\hat{g}(\omega)}$ was not used in the verification of (8).

It remains to show that (9) is equal to zero. Fix $\alpha \in \mathbb{Z} \backslash 2 \mathbb{Z}$. By a change of variables $\gamma \mapsto-\gamma^{\prime}+\alpha$, we obtain

$$
t_{\alpha, \mathcal{W}}(\omega)=\sum_{\gamma^{\prime} \in \mathbb{Z}}(-1)^{\left|\left(-\gamma^{\prime}+\alpha\right)\right|} \hat{g}\left(\omega+\gamma^{\prime}-\alpha\right) \overline{\hat{g}\left(\omega-\gamma^{\prime}\right)}
$$

for a.e. $\omega \in \mathbb{R}$. For odd $\alpha \in \mathbb{Z}$, we note that

$$
(-1)^{\left|-\gamma^{\prime}+\alpha\right|}=-(-1)^{\left|\gamma^{\prime}\right|} .
$$

By our assumption $\hat{g}(\omega)=\overline{\hat{g}(\omega)}$, it follows that

$$
\hat{g}\left(\omega+\gamma^{\prime}-\alpha\right) \overline{\hat{g}\left(\omega-\gamma^{\prime}\right)}=\overline{\hat{g}\left(\omega+\gamma^{\prime}-\alpha\right)} \hat{g}\left(\omega-\gamma^{\prime}\right) .
$$

From the last three displayed equations, we see that $t_{\alpha, \mathcal{W}}(\omega)=-t_{\alpha, \mathcal{W}}(\omega)$, hence $t_{\alpha, \mathcal{W}}(\omega)=0$.

Lemma III.3 shows a very simple relationship between the autocorrelation functions of Gabor systems and Wilson systems. In fact:

$$
t_{\alpha, \mathcal{W}}(\omega)= \begin{cases}2^{-1} t_{\alpha, \mathcal{G}}(\omega) & \alpha \in \Lambda^{\perp} \\ 0 & \alpha \in \mathbb{Z}^{d} \backslash\left(\Lambda^{\perp}\right) .\end{cases}
$$

\section{A proof of $S_{\mathcal{G}}=2 S_{\mathcal{W}}$}

To illustrate the usefulness of Lemma III.3, we give a simple proof of $S_{\mathcal{G}}=2 S_{\mathcal{W}}$.

Proof of Proposition II.2. We only argue for the proof of the "furthermore"-part, i.e., (2). The Walnut representations of the two frame operators show that

$$
\begin{aligned}
\widehat{S_{\mathcal{W} f}}=\sum_{\alpha \in \mathbb{Z}^{d}} M_{t_{\alpha, \mathcal{W}}} T_{\alpha} \hat{f} \\
=2^{-1} \sum_{\alpha \in \Lambda^{\perp}} M_{t_{\alpha, \mathcal{G}}} T_{\alpha} \hat{f}=2^{-1} \widehat{S_{\mathcal{G}} f}
\end{aligned}
$$

for $f$ in a dense subset of $L^{2}\left(\mathbb{R}^{d}\right)$. By continuity the relation extends to all of $L^{2}\left(\mathbb{R}^{d}\right)$.

\section{EXTENSIONS}

It is possible to extend the results from Section II on multivariate Wilson bases in several directions. Firstly, it is possible to construct multivariate Wilson bases on symplectic lattices. In dimension one this result generalizes a result by Kutyniok and Strohmer [8]. Secondly, it is possible to define a huge number of distinct intermediate $2^{k}$-modular Wilson systems for $k=1, \ldots, d-1$. We refer the reader to [10] for the precise statements.

\section{REFERENCES}

[1] R. Balian. Un principe d'incertitude fort en théorie du signal ou en mécanique quantique. C. R. Acad. Sci. Paris, 292(20):13571362, 1981.

[2] J. J. Benedetto, C. Heil, and D. F. Walnut. Differentiation and the Balian-Low theorem. J. Fourier Anal. Appl., 1(4):355-402, 1995.

[3] O. Christensen. An introduction to frames and Riesz bases. Applied and Numerical Harmonic Analysis. Birkhäuser Boston Inc., Boston, MA, 2003.

[4] I. Daubechies, S. Jaffard, and J.-L. Journé. A simple Wilson orthonormal basis with exponential decay. SIAM J. Math. Anal., 22(2):554-573, 1991.

[5] K. Gröchenig. Foundations of time-frequency analysis. Applied and Numerical Harmonic Analysis. Birkhäuser Boston, Inc., Boston, MA, 2001.

[6] E. Hernández, D. Labate, and G. Weiss. A unified characterization of reproducing systems generated by a finite family. II. J. Geom. Anal., 12(4):615-662, 2002.

[7] A. J. E. M. Janssen. The duality condition for Weyl-Heisenberg frames. In Gabor analysis and algorithms, Appl. Numer. Harmon. Anal., pages 33-84. Birkhäuser Boston, Boston, MA, 1998.

[8] G. Kutyniok and T. Strohmer. Wilson bases for general time-frequency lattices. SIAM J. Math. Anal., 37(3):685-711 (electronic), 2005.

[9] F. Low. Complete sets of wave packets. In C. D. et al., editor, $A$ passion for Physics-Essays in Honor of Geoffrey Chew, pages 17-22. World Scientific, Singapore, 1985.

[10] M. Bownik, M. S. Jakobsen, J. Lemvig, and K. A. Okoudjou. On Wilson bases in $L^{2}\left(\mathbb{R}^{d}\right)$. Preprint, arXiv:1704.06510, 2017.

[11] K. G. Wilson. Generalized Wannier functions. 1987. unpublished manuscript. 